\title{
Hospital-acquired bloodstream infections in neurological and neurosurgical units in Hungary, 2005-2013
}

\author{
R Szabó ${ }^{*}$ A Kurcz
}

From 3rd International Conference on Prevention and Infection Control (ICPIC 2015)

Geneva, Switzerland. 16-19 June 2015

\section{Introduction}

Hospital-acquired bloodstream infection (HABSI) is a serious complication of hospitalization with high associated morbidity and mortality, within and outside of the intensive care units. However, information related to HABSIs among neurological and neurosurgical patients are limited.

\section{Objectives}

Our objective was to describe the epidemiological trends of HABSIs in this patient population in Hungary performed a nine-year descriptive epidemiological analysis.

\section{Methods}

Patient data were recorded and submitted into the national surveillance database (National Nosocomial Surveillance system) by the local infection control practitioners from the reporting hospitals. Based on this database, descriptive statistics were performed using EpiData version 3.1 (http://www.epidata.dk).

\section{Results}

The overall incidence rate was 0.4 HABSIs per 100 discharges in both ward types. HABSIs were considered primary in $66.8 \%$ among neurological patients and $58.9 \%$ among neurosurgical patients. For secondary HABSIs, the primary infection site was respiratory tract infection (19.5\% and $17.8 \%)$ in both ward type. The most common pathogen was the Staphylococcus aureus (19.3\%) in neurological wards and the coagulase-negative staphylococci $(17.4 \%)$ in neurosurgical wards. The overall case fatality rate was $9.2 \%$.

Department of Hospital Epidemiology and Hygiene, National Center for Epidemiology, Budapest, Hungary 